\title{
Imaging of temperature dependent hemodynamics in the rat sciatic nerve by functional photoacoustic microscopy
}

Lun-De Liao', Josue Orellana', Yu-Hang Liu', Yan-Ren Lin' ${ }^{3,4}$, Ashwati Vipin ${ }^{1}$, Nitish V Thakor ${ }^{1,2}$, Kaiquan Shen ${ }^{1 *}$ and Einar Wilder-Smith ${ }^{1,5^{*}}$

\footnotetext{
* Correspondence: kqshen@gmail. com; einar_wilder-smith@nuhs.edu.sg ${ }^{1}$ Singapore Institute for Neurotechnology (SINAPSE), National University of Singapore, 28 Medical Drive, \#05-COR, Singapore 117456, Singapore ${ }^{5}$ Department of Medicine, Yong Loo Lin School of Medicine, National University of Singapore, Singapore 117456, Singapore

Full list of author information is available at the end of the article
}

\begin{abstract}
Background: Vascular hemodynamics is central to the regulation of neuro-metabolism and plays important roles in peripheral nerves diseases and their prevention. However, at present there are only a few techniques capable of directly measuring peripheral nerve vascular hemodynamics.
\end{abstract}

Method: Here, we investigate the use of dark-field functional photoacoustic microscopy (fPAM) for intrinsic visualizing of the relative hemodynamics of the rat sciatic nerve in response to localized temperature modulation (i.e., cooling and rewarming).

Results and conclusion: Our main results show that the relative functional total hemoglobin concentration $(\mathrm{HbT})$ is more significantly correlated with localized temperature changes than the hemoglobin oxygen saturation $\left(\mathrm{SO}_{2}\right)$ changes in the sciatic nerve. Our study also indicates that the relative $\mathrm{HbT}$ changes are better markers of neuronal activation than $\mathrm{SO}_{2}$ during nerve temperature changes. Our results show that fPAM is a promising candidate for in vivo imaging of peripheral nerve hemodynamics without the use of contrast agents. Additionally, this technique may shed light on the neuroprotective effect of hypothermia on peripheral nerves by visualizing their intrinsic hemodynamics.

Keywords: Peripheral nerve imaging, Functional photoacoustic microscopy (fPAM), Temperature stimulation, Total hemoglobin concentration $(\mathrm{HbT})$, Hemoglobin oxygen saturation $\left(\mathrm{SO}_{2}\right)$

\section{() Biomed Central}

\section{Introduction}

Visualizing hemodynamic changes through total hemoglobin concentration (HbT) and hemoglobin oxygen saturation $\left(\mathrm{SO}_{2}\right)$ can lead to high impact in vivo research and clinical applications in the treatment and prevention of peripheral nerve diseases [1-5]. These include the study of thermoregulatory sensory pathways $[6,7]$, peripheral nerve injury $[3,8,9]$ and disease models such as neuropathy $[1,10]$. Medical ultrasound imaging (MUI) and functional magnetic resonance imaging (fMRI) are the two most prominent techniques for imaging the structure and function of peripheral nerve, respectively [3]. MUI provides real-time blood flow and morphological information of the nerve. Liu et al. applied high-frequency MUI to evaluate morphological changes in human diabetic neuropathy by visualizing the relative change in the fascicular size of

(c) 2013 Liao et al.; licensee BioMed Central Ltd. This is an open access article distributed under the terms of the Creative Commons Attribution License (http://creativecommons.org/licenses/by/2.0), which permits unrestricted use, distribution, and reproduction in any medium, provided the original work is properly cited. 
sural nerves [3]. Moreover, Jan et al. used laser Doppler to investigate the skin blood flow response to locally applied mechanical and thermal stresses for the study of diabetic neuropathy [11]. However, label-free bio-tissue imaging of physiological parameters (i.e., $\mathrm{SO}_{2}$ ) using MUI or laser Doppler is still challenging [3,11]. In contrast, fMRI maps hemodynamics by measuring surrogate changes in blood flow and oxygenation, which accompany changes in neural activity. Probing the hemodynamics down to the level of a single nerve group and its nurturing blood vessels without the use of contrast agents is difficult to achieve for most of other imaging techniques, including fMRI [12].

Recently, optical imaging has been increasingly used to study hemodynamics in vivo $[13,14]$; the optical spectroscopy method can probe oxy- and deoxy-hemoglobin (i.e., $\mathrm{HbO}_{2}$ and $\mathrm{Hb}$ ) through its distinct optical absorption characteristics [13,15-17]. But, due to light's limited penetration depth $[14,18]$, optical imaging techniques can only probe information from the surface level of target blood vessels [19-21] and provide no structural information for the deeper tissue [14,22]. Though current MUI, laser Doppler, fMRI and optical imaging techniques can register vascular responses by using their intrinsic contrast, the capability of label free study of in vivo hemodynamics at the peripheral nerve level is still beyond their conventional use [18].

Photoacoustic (PA) is a novel optical absorption-based hybrid imaging methodology that combines the merits of ultrasound and optical imaging techniques and allows for the monitoring of intrinsic hemodynamic changes with a deep penetration reach $[23,24]$ and without the use of contrast agents. PA imaging technique has three major implementations: dark-field PA microscopy (PAM), PA computed tomography (PAT) and PA endoscopy (PAE). Whereas PAM and PAE usually aim to image millimeters deep at micrometer-scale resolution, PAT can be implemented for deeper imaging ability but only with few hundred micrometer-scale resolution. A reflection-mode functional PAM (fPAM) has been applied to subcutaneous vasculature [24], tumor detection of breast [25] and brain [26], oxygenation monitoring in blood vessels and neurovascular imaging $[23,27,28]$ in small animal models.

Here, we report dark-field fPAM as a reliable imaging technique for the label-free study of in vivo relative hemodynamics in the rat sciatic nerve during temperature modulation via cooling and rewarming. The fundamental thermoregulatory implications of localized nerve temperature changes have not been fully elucidated [8]. For the first time, we demonstrate this methodology for in vivo visualization of the relative hemodynamics in the rat sciatic nerve. We studied and characterized the temperature dependent vascular dynamics of the rat sciatic nerve under targeted cooling and rewarming conditions. This methodology has significant potential for enhancing our understanding of nerve hemodynamics as well as providing novel insights for evaluating the vascular environment under various stimulation conditions, especially involving peripheral nerve diseases in small animal models [29].

\section{Materials and methods}

\section{Dark-field confocal functional photoacoustic microscopy system}

Our 50-MHz dark-field confocal fPAM system for imaging functional hemodynamics in the sciatic nerve is shown in Figure 1 consisting of laser pulse generation and delivery (Figure 1A), PA signal reception, and image reconstruction and display (Figure 1B). Laser pulses, $4 \mathrm{~ns}$ wide, were generated at a frequency of $10 \mathrm{~Hz}$ by using an optical 


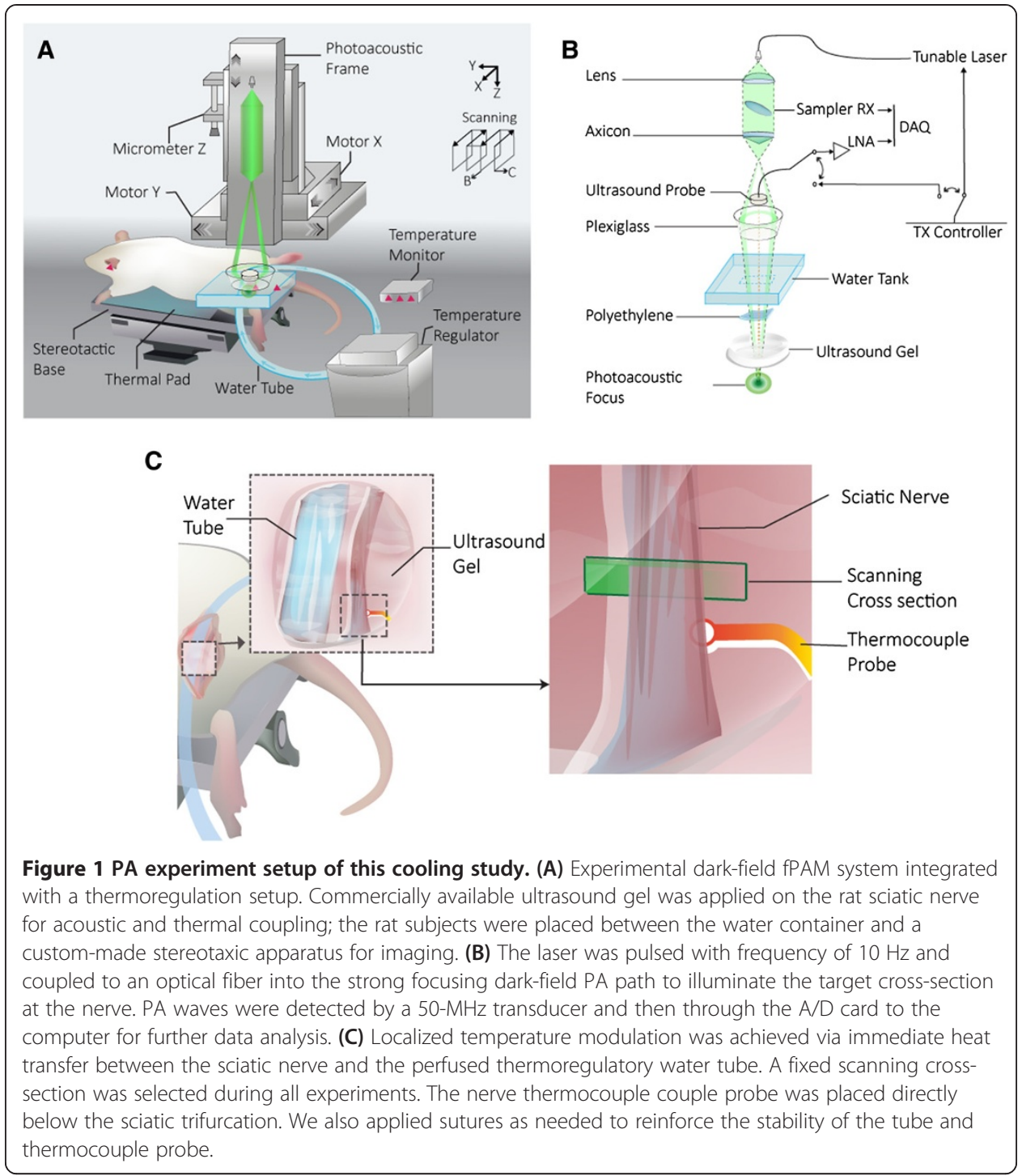

parametric oscillator (Surlite OPO Plus, Continuum, USA). The laser was pumped by a frequency-tripled Nd:YAG Q-switched laser (Surlite II-10, Continuum, USA). Two visible wavelengths of the laser pulses, 560 and $570 \mathrm{~nm}\left(\lambda_{560}\right.$ and $\left.\lambda_{570}\right)$, were employed for PA wave excitation [27]. At the selected wavelengths, blood is a dominant optical absorber, producing strong optical absorption and thus guaranteeing that the detected PA signals mainly come from blood [23,27]. The acquired PA signal at $\lambda_{560}$ is sensitive to relative changes in $\mathrm{SO}_{2}$, while relative $\mathrm{HbT}$ changes are the most prominent at $\lambda_{570}$ [23]. The $50-\mathrm{MHz}$ ultrasonic transducer used in the current fPAM system was custommade by the Acoustic Sensor Co., Ltd at Taiwan. It has a $-6 \mathrm{~dB}$ fractional bandwidth of $57.5 \%$, a focal length of $9 \mathrm{~mm}$ and a $6 \mathrm{~mm}$ active element, offering an axial resolution of $32 \mu \mathrm{m}$ and a lateral resolution of $61 \mu \mathrm{m}$.

Laser energy was delivered using a 1-mm multimodal fiber (Thorlabs, U.S.A). The fiber tip was coaxially aligned with a convex lens, an axicon, a plexiglass mirror, and an ultrasonic transducer on an optical bench, forming dark-field illumination that was 
confocal with the focal point of the ultrasonic transducer. The incident energy density on the sample surface was well within American National Standards institute (ANSI) safety limits. The transducer was immersed in an acrylic water tank during the imaging process, and the hole at the bottom of the tank was sealed with a piece of $15-\mu \mathrm{m}$ thick polyethylene film. A thin layer of ultrasonic gel was applied as a PA and thermal conductive medium, which was then attached to the thin polyethylene film to ensure reliable coupling of the PA waves with the water tank. The PA signals received by the ultrasonic transducer were pre-amplified by a low-noise amplifier (noise figure $1.2 \mathrm{~dB}$, gain $55 \mathrm{~dB}, \mathrm{AU}-3 \mathrm{~A}-0110$, USA), cascaded to an ultrasonic receiver (5073 PR, Olympus, USA) and then digitized and sampled by a computer-based 14-bit analog to digital (A/D) card (CompuScope 14220, GaGe, USA) at a $200-\mathrm{MHz}$ sampling rate for data storage.

Fluctuations in the laser energy were monitored with a photodiode (DET36A/M, Thorlabs, USA). The recorded photodiode signals were measured prior to the experiment to compensate for PA signal variations caused by laser-energy instability. The achievable penetration depth of the current fPAM system was $3 \mathrm{~mm}$ with approximately 18-dB SNR, where SNR is defined as the ratio of the signal peak value to the root-mean-square value of the noise. Three scan types can be provided by this system (Figure 1A): A-line (i.e., one-dimensional images where the axis represents the imaging depth), B-scan (i.e., two-dimensional images where one axis is the lateral scanning distance and the other is the imaging depth), and C-scan (i.e., projection images from the three-dimensional images) [24]. The amplitude of the envelope-detected PA signals was used in the subsequent functional imaging analysis [23].

\section{Experimental animals}

Ten adult female Wistar rats (NUS-CARE, Singapore) weighing $280 \pm 20$ grams were used and housed at a constant temperature and humidity with free access to food and water. The Institutional Animal Care and Use Committee (IACUC) at the National University of Singapore approved all the experimental procedures.

Rats remained anesthetized with isoflurane $2-3 \%$ in $100 \% \mathrm{O}_{2}$ and were mounted on a dorsal position over a custom-made acrylic stereotaxic holder. Next, the left hind limb was shaved and disinfected prior to making a $40 \mathrm{~mm}$ longitudinal incision at knee level. The biceps femoris was detached and folded towards the posterior. Also the caudofemoralis was transected in order to completely expose the sciatic nerve [30].

A thermoregulatory device was customized to provide localized temperature modulation to the exposed rat sciatic nerve. The apparatus consisted of a flexible tube (Bev-ALine IV, out diameter at $4.8 \mathrm{~mm}$, inner diameter at $3.2 \mathrm{~mm}$, thermoplastic processes, $\mathrm{NJ}$, USA), which was inserted through $5 \mathrm{~mm}$ sub-muscular incisions and placed in parallel at about $5 \mathrm{~mm}$ to the left of the in situ sciatic nerve as shown in Figure 1C.

\section{Thermoregulation}

Temperature controlled water was continually circulated through the tube for immediate cooling and subsequent rewarming of the sciatic nerve using Blanketrol II system (Cincinnati Sub-zero, OH, USA), as shown in Figure 1A and 1B. A thermal blanket was placed on the ventral surface of the animal in order to maintain core body temperature 
at normothermia $\left(37 \pm 0.5^{\circ} \mathrm{C}\right)$. Thermocouple probes were used to monitor tympanic, rectal and sciatic nerve temperatures (prior to trifurcation) (as indicated in Figure 1A and $1 \mathrm{C}$ ). The temperature data were recorded at $2 \mathrm{~Hz}$ using Thermes USB acquisition system and proprietary software (Physitemp, NJ, USA).

The temperature modulation protocol consisted of three stages: baseline, cooling and rewarming for all experimental animals (Figure 2D). Baseline stage was recorded while the sciatic nerve temperature remained at $33 \pm 2^{\circ} \mathrm{C}$. The cooling stage began 20 minutes after the onset of baseline recording. At this point, the sciatic nerve temperature was monotonically decreased by at least $10^{\circ} \mathrm{C}$ at an approximate rate of $-0.5^{\circ} \mathrm{C} / \mathrm{min}$. The cooling period ended after a plateau was maintained for 40 minutes. Subsequently, the sciatic nerve temperature was reverted to its baseline target during the rewarming stage, with a monotonic increase of about $0.5^{\circ} \mathrm{C} / \mathrm{min}$. The experiment concluded after 20 minutes of a maintained rewarmed state.

\section{Data analysis of the functional changes in $\mathrm{HbT}$ and $\mathrm{SO}_{2}$}

Two optimized wavelengths (i.e., $\lambda_{560}$ and $\lambda_{570}$ ) were employed for monitoring the functional $\mathrm{HbT}$ and $\mathrm{SO}_{2}$ changes with a high SNR and sensitivity [23]. The optical absorption of blood at $\lambda_{560}$ is sensitive to $\mathrm{SO}_{2}$ levels, while the blood absorption at $\lambda_{570}$ results from the isobestic point of molar extinction spectra for oxy- and deoxyhemoglobin $[18,31]$. Because the $\lambda_{570}$ PA signal at a given pixel is proportional to the

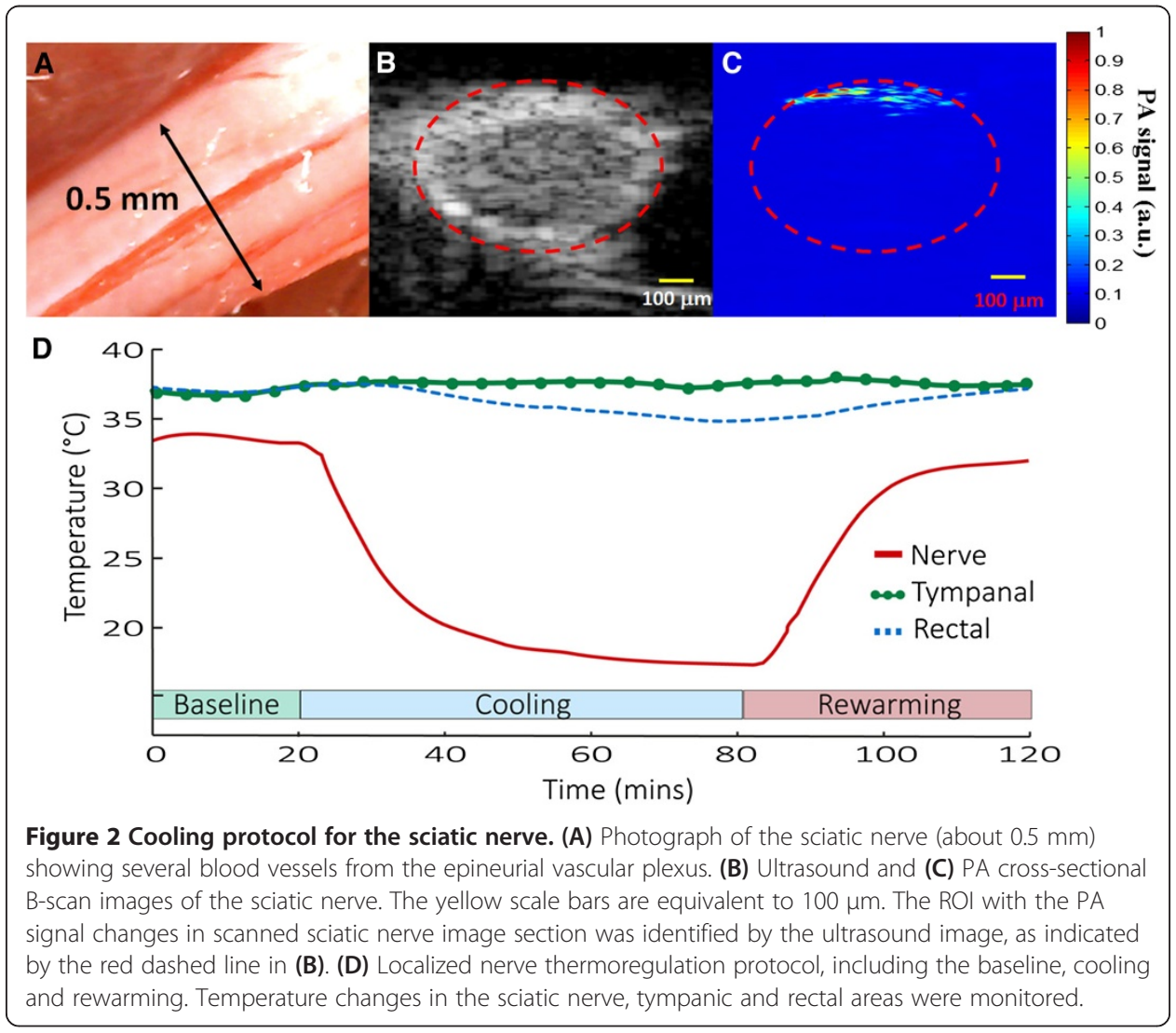


$\mathrm{HbT}$ within its resolution cell centered at that pixel, the mean functional $\mathrm{HbT}$ changes $\left(R_{\mathrm{HbT}}(t)\right)$ in the selected sciatic nerve region can be assessed as follows:

$$
R_{\mathrm{HbT}}(t)=\frac{\sum_{(\mathrm{x}, \mathrm{z}) \in \mathrm{ROI} \text { pixel }}\left(I_{(570)}(\mathrm{x}, \mathrm{z}, t)\right) / A\left(I_{(570)}\left(t_{0}\right)\right)}{\sum_{(\mathrm{x}, \mathrm{z}) \in \mathrm{ROI} \text { pixel }}\left(I_{(570)}\left(\mathrm{x}, \mathrm{z}, t_{0}\right)\right) / A\left(I_{(570)}\left(t_{0}\right)\right)} \times 100 \%,
$$

where $(\mathrm{x}, \mathrm{z})$ is the pixel position; $I_{(570)}(\mathrm{x}, \mathrm{z}, t)$ is the PA image at $\lambda_{570}$ acquired at time $t$ and $I_{(570)}\left(\mathrm{x}, \mathrm{z}, t_{0}\right)$ is the baseline PA signal at $\lambda_{570}$ acquired immediately before the onset of cooling (i.e. at the baseline $\left.t_{0}\right) ; A\left(I_{(570)}\left(t_{0}\right)\right)$ represents the total pixel count of regions of interest (ROI) at the baseline $t_{0}$ [18]. Here, the ROI pixel was defined as the pixel that possessed a PA signal that was at least three times greater than the background signal $[23,32]$.

Functional images of $\mathrm{SO}_{2}$ changes $\left(I_{F(560)}(t)\right)$ at a given time point, $t$, at each stage were assessed according to the following equation:

$$
I_{F(560)}(t)=\frac{I_{(560)}(t)}{I_{(570)}(t)}
$$

where $I_{(560)}(t)$, i.e., PA image acquired at $\lambda_{560}$, was normalized to $I_{(570)}(t)$ on a pixel-bypixel basis [18]. The mean functional $\mathrm{SO}_{2}$ changes $\left(R_{\mathrm{SO}_{2}}(t)\right)$ in a single ROI region during the stimulation period were probed as follows:

$$
R_{\mathrm{SO}}(t)=\frac{\sum_{(\mathrm{x}, \mathrm{z}) \in \mathrm{ROI} \text { pixel }}\left(I_{F(560)}(\mathrm{x}, \mathrm{z}, t)\right) / A\left(I_{(570)}\left(t_{0}\right)\right)}{\sum_{(\mathrm{x}, \mathrm{z}) \in \mathrm{ROI} \text { pixel }}\left(I_{F(570)}\left(\mathrm{x}, \mathrm{z}, t_{0}\right)\right) / A\left(I_{(570)}\left(t_{0}\right)\right)} \times 100 \% .
$$

That is, an independent probing of the changes in $\mathrm{HbT}$ and $\mathrm{SO}_{2}$ could be achieved where $I_{(570)}$ was used as a marker for $\mathrm{HbT}$, and $I_{F(560)}$ was used as a marker for $\mathrm{SO}_{2}[18,23,33]$.

This experiment was designed to quantitatively compare the differences in relative PA signal changes from temperature modulation in the vasculature of the rat sciatic nerve. In the current PPAM setting, the data acquisition time for each PA B-scan image with 31 scanned lines ( $2 \mathrm{~mm}$ width) is about 28 seconds. Hence, it takes about 56 seconds for one functional image of $I_{F(560)}$. To identify vascular changes in response to temperature modulation, functional ultrasound and PA images were registered at a fixed cross-sectional area. Images acquired from the ultrasound scanning of the sciatic nerve were used as a reference to identify morphological characteristics and the PA region of interest (ROI) as indicated by the red dashed line in Figure $2 \mathrm{~B}$ and $2 \mathrm{C}$.

Statistical significance was assessed using a paired $t$-test with significance defined as $p$-value of $<0.05$ for the side-to-side differences in PA signals $\left(I_{R(570)}\right.$ and $\left.I_{F(560)}\right)$ of the studied areas. The significance of changes observed in fPAM signals $\left(I_{F(560)}\right)$ at the respective ROI in response to temperature modulation was compared using the Wilcoxon matched-pairs signed-rank test (two-tailed, $p<0.05, n=10$ ) [23,27,33]. All statistical analyses were performed using SPSS (version 10.0, SPSS ${ }^{\circ}$, USA). 


\section{Results}

\section{PA imaging of the rat sciatic nerve vasculature}

A photograph of the surface of rat sciatic nerve is shown in Figure 2A. Many distinct blood vessels varying in size can be seen at the epineurium of the sciatic nerve. The $\mathrm{B}$-scan ultrasound and PA images of the rat sciatic nerve are shown in Figure $2 \mathrm{~B}$ and $2 \mathrm{C}$, respectively. Increased relative $\mathrm{HbT}$ and $\mathrm{SO}_{2}$ values peak in the same areas that the blood vessel are seen, suggesting that these regions represent blood vessels as visualized by fPAM (Figure 2C).

\section{Localized thermoregulation response}

A selected cross-section region of the sciatic nerve was examined for relative side-toside PA signal differences in response to localized temperature modulation. The thermoregulation protocol was designed to quantitatively compare the relative $\mathrm{HbT}$ and $\mathrm{SO}_{2}$ changes in vascular structures of the sciatic nerve between three localized temperature modulation stages: baseline, cooling and rewarming (Figure 2D). Figure 3A and Additional file 1: Movie S1 show the response for both relative HbT changes as function of time under the thermoregulation protocol.

\section{Functional temperature dependent hemodynamics in sciatic nerve}

In vivo functional hemodynamics of the sciatic nerve in response to thermoregulation at difference stages are shown in Figure 3A. The ultrasound and PA images are shown in Figure $2 \mathrm{~B}$ and $2 \mathrm{C}$, respectively, and their ROIs were used for the statistical analysis. Both relative $\mathrm{HbT}$ and $\mathrm{SO}_{2}$ changes demonstrate significant correlations with localized

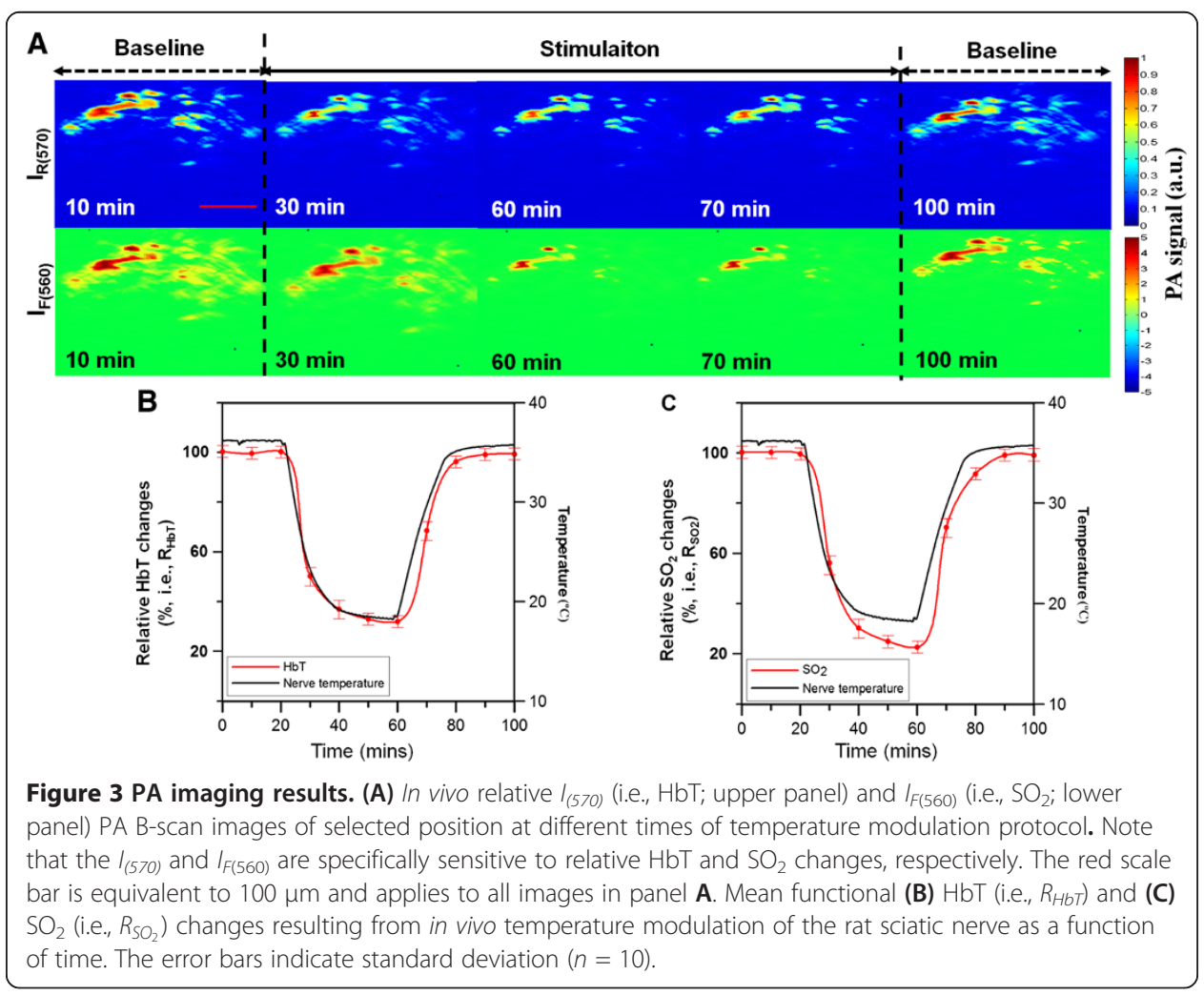


thermoregulation during cooling and rewarming stages, as shown in Figure 3B and 3C, respectively ( $p<0.05$; paired $t$-test). Quantitative analysis shows that during cooling, relative $\mathrm{HbT}\left(R_{H b T}\right)$ was reduced by $-70.8 \%$ with respect to its baseline, and correlated with temperature by $\mathrm{r}=0.94$. Similarly, relative $\mathrm{SO}_{2}\left(\mathrm{R}_{\mathrm{SO}_{2}}\right)$ was lowered by $-73.3 \%$ from its baseline value, and correlated with temperature by $r=0.88$. The applied thermoregulation protocol did not significantly affect $R_{\mathrm{HbT}}$ and $R_{\mathrm{SO}_{2}}$ in being restored to its initial baseline values ( $p>0.05$; paired $t$-test).

\section{Discussion}

The present study used the fPAM technique to investigate the relative $\mathrm{HbT}$ and $\mathrm{SO}_{2}$ changes in a specific cross-section region of the rat sciatic nerve in response to temperature modulation by cooling and rewarming. Our main findings are as follows:

1) The functional $\mathrm{HbT}$ and $\mathrm{SO}_{2}$ changes in the rat sciatic nerve can be reliably investigated in a label-free manner using the described fPAM settings.

2) The functional $\mathrm{HbT}$ and $\mathrm{SO}_{2}$ changes were linearly correlated with temperature in both cooling and rewarming stages.

3) For the first time, the results suggest that relative $\mathrm{HbT}$ has a stronger correlation with localized nerve temperature changes in comparison to $\mathrm{SO}_{2}$ changes within the dynamic range of temperature modulation tested in this study.

\section{Temperature dependent vasculature hemodynamics in rat sciatic nerve}

As shown in our experimented results, the presented fPAM setting is able to directly measure the relative $\mathrm{HbT}$ and $\mathrm{SO}_{2}$ changes at $32 \times 61 \mu \mathrm{m}$ resolution in an exposed nerve. This level of measurement cannot be directly and reliably detected by other existing imaging techniques [18,34]. fPAM can independently measure hemodynamic features according to the intrinsic optical absorption spectra $[17,18]$, where $I_{(570)}$ is used as a marker of relative HbT changes, and $I_{F(560)}$ as a marker of relative $\mathrm{SO}_{2}$ changes [23].

Our findings show that both $R_{H b T}$ and $R_{S_{2}}$ in a selected ROI of the sciatic nerve decreased significantly during the cooling stage (Figure 3). Both changes in $\mathrm{HbT}$ and $\mathrm{SO}_{2}$ were significantly proportional to temperature changes (Figure 3) [35,36]. But it is also noted that $R_{\mathrm{SO}_{2}}$ decreased to a lower relative value when compared to $R_{H b T}$ and in response to cooling by about $10^{\circ} \mathrm{C}$. These results correlate well with previous laser Doppler flowmetry studies [9,37] showing the clear relationship between the blood flow and tissue temperature [38]. It is intriguing to observe $R_{H b T}$ being more strongly correlated with temperature modulation than $\mathrm{RSO}_{2}$ (Figure 3B and $3 \mathrm{C}$ ). One plausible explanation is that there are two major effects resulting from nerve temperature changes: 1) vasoconstriction due to lowered temperatures and 2) metabolism down-regulation due to reduced temperature levels. A dominant effect of vasoconstriction over metabolism downregulation results in more significant relative HbT change [39,40]. Nemoto et al. measured cortical evoked responses to somatosensory stimuli and showed that HbT-related signals are more highly correlated to the local region than oxygenation-related signals [41].

Characteristics of the current functional PAM system compared to other imaging techniques MUI is a useful method, which provides satisfactory indicators of morphological and blood flow changes [3], even in the nerve. But, MUI is limited by not being able to 
utilize intrinsic contrast for measurements of hemodynamic parameters. However, the relationship between morphological changes and their hemodynamics plays an important role for developing a better understanding of neurovascular functions. Thus, the development of the current fPAM system can be of significant benefit for the interpretation of MUI by registering intrinsic hemodynamic signals. Blood oxygen-level dependent (BOLD) fMRI is another method used to study hemodynamics, but it lacks selectivity between oxygen delivery and consumption, which may both occur during neuronal activation [42]. For instance, it is possible for a negative BOLD signal to occur under very high neuronal activity, where changes in oxygen consumption may far exceed oxygen supply [43]. Indeed, the interpretation of a given BOLD signal response may be more accurate $[23,33]$ by combining it with fPAM to isolate the contribution of $\mathrm{SO}_{2}$ changes.

The $50 \mathrm{MHz}$ ultrasound B-scan image provides a view of the complete cross-section of the sciatic nerve (Figure 2B), but only a part of it can be imaged by fPAM. This discrepancy is caused by the penetration depth of light in body tissue and blood [16,17]. Limited penetration depth of light in blood vessels restricts the maximum measurable axial diameter registered by the current fPAM system [33] as shown in (Figure 2C). To an extent, the dark-field illumination employed in PPAM system and the natural scattering of light in nerve tissue helps to increase the penetration of light in bio-tissue in this in vivo experiments [33]. The scope of this study includes only the temperature dependent hemodynamics visualized with fPAM for a ROI (Figure 3A). We would like to emphasize, that presently we are only considering relative and micro-resolution functional hemodynamic changes in the blood vessels of rat sciatic nerve, as opposed to absolute vasculature changes.

While there has been a lot interest in studying hemodynamics or neurovascular functions as well as the imaging of such coupling in relation to therapies such as hypothermia in the brain, very little has been done to study the neurovascular coupling in the peripheral nerves [44]. We aim to understand and develop the in vivo fPAM technique for the study of peripheral nervous system hemodynamics. The peripheral nerve system presents itself an ideal object of study since the nerves lie just millimeters below the skin, providing a simple and robust model to study hemodynamics in response to temperature changes.

Here, we report fundamental correlations between relative $\mathrm{HbT}$ and $\mathrm{SO}_{2}$ in response to temperature modulation via cooling and rewarming. Further investigation is needed to better understand the relationship between hemodynamics and neuronal activation under different modalities of temperature stimulation. In addition to temperature modulation of the nerve, it is worth mentioning that this technique may also be applicable to monitor the effects of various other in vivo stimulation protocols, such as electrical [23] and pharmacological. Most importantly we envisage this technique to be useful for determining blood flow dynamics in human neuropathies caused by vascular pathology such as diabetic neuropathy [11].

\section{Conclusions}

In summary, our results show that the current PPAM technique is a good candidate for detecting the hemodynamic changes reflected in the relative $\mathrm{HbT}$ and $\mathrm{SO}_{2}$ fluctuations evoked by temperature changes in rat peripheral nerve. We found that changes in 
relative $\mathrm{HbT}$ are more linearly correlated to nerve temperature modulation than $\mathrm{SO}_{2}$. This finding suggests that the significant relative $\mathrm{HbT}$ changes are more co-localized with neuronal activation in the nerve. We also show that the regulation of sciatic nerve hemodynamics can be reliably studied by fPAM technique without the use of contrast agents. The technique we describe may help shed light on the neuroprotective effect of hypothermia on peripheral nerve by visualizing its intrinsic hemodynamics. Along this line, it may also add to our understanding of peripheral nerve hemodynamics and present important implications for the development of new therapeutic approaches.

\title{
Additional file
}

Additional file 1: Movie S1. This movie shows the response for both relative HbT changes as function of time under the thermoregulation protocol (i.e., cooling and rewarming stages).

\begin{abstract}
Abbreviations
fPAM: Functional photoacoustic microscopy; PA: Photoacoustic; HbT: Total hemoglobin concentration; $\mathrm{SO}_{2}$ : Hemoglobin oxygen saturation; MUI: Medical ultrasound imaging; fMRI: Functional magnetic resonance imaging; $\mathrm{HbO}_{2}$ : Oxy-hemoglobin; $\mathrm{Hb}$ : Deoxy-hemoglobin; SNR: Ratio of the signal peak value to the root-mean-square value of the noise; ANSI: American National Standards institute; IACUC: Institutional Animal Care and Use Committee; ROI: Region of interest; BOLD: Blood oxygen-level dependent.

Competing interests

The authors declared that they have no competing interests.

\section{Authors' contributions}

Study concept and design (LDL, JO, KQS and EWS); drafting of the manuscript (LDL and JO); critical revision of the manuscript for important intellectual content (LDL, JO, AV, YHL, NT, KQS and EWS); obtained funding (NT, LDL); administrative, technical, and material support (LDL, JO, NT, KQS and EWS); study supervision (LDL, NT, AL, KQS and EWS). All authors read and approved the final manuscript.
\end{abstract}

\section{Acknowledgement}

The authors thank the National University of Singapore for supporting the Neurovascular Imaging Lab at the Singapore Institute for Neurotechnology (SINAPSE) under grant number R-711-000-026-133.

\section{Author details}

${ }^{1}$ Singapore Institute for Neurotechnology (SINAPSE), National University of Singapore, 28 Medical Drive, \#05-COR, Singapore 117456, Singapore. ${ }^{2}$ Department of Biomedical Engineering, Johns Hopkins University, Traylor 701/720 Rutland Ave, Baltimore 21205, USA. ${ }^{3}$ Department of Emergency Medicine, Changhua Christian Hospital, Changhua, Taiwan. ${ }^{4}$ School of Medicine, Chung Shan Medical University, Taichung, Taiwan. ${ }^{5}$ Department of Medicine, Yong Loo Lin School of Medicine, National University of Singapore, Singapore 117456, Singapore.

Received: 26 August 2013 Accepted: 9 October 2013

Published: 18 November 2013

\section{References}

1. Bruno RM, Ghiadoni L, Seravalle G, Dell'oro R, Taddei S, Grassi G: Sympathetic regulation of vascular function in health and disease. Front Physiol 2012, 3:284.

2. Kluding PM, Pasnoor M, Singh R, Jernigan S, Farmer K, Rucker J, Sharma NK, Wright DE: The effect of exercise on neuropathic symptoms, nerve function, and cutaneous innervation in people with diabetic peripheral neuropathy. J Diabetes Complications 2012, 26:424-429.

3. Liu F, Zhu JA, Wei M, Bao YQ, Hu B: Preliminary evaluation of the sural nerve using 22-MHz ultrasound: a new approach for evaluation of diabetic cutaneous neuropathy. PLOS ONE 2012, 7(4):e32730.

4. Arrich J, Holzer M, Havel C, Mullner M, Herkner H: Hypothermia for neuroprotection in adults after cardiopulmonary resuscitation. Cochrane Database Syst Rev 2012, 9, CD004128.

5. Soleimani M, Shipley RJ, Smith N, Mitchell CN: Medical imaging and physiological modelling: linking physics and biology. Biomed Eng Online 2009, 8:1.

6. Nakamura K, Morrison SF: A thermosensory pathway that controls body temperature. Nat Neurosci 2008, 11:62-71.

7. Koganezawa T, Ishikawa T, Fujita Y, Yamashita T, Tajima T, Honda M, Nakayama K: Local regulation of skin blood flow during cooling involving presynaptic P2 purinoceptors in rats. Br J Pharmacol 2006, 148:579-586.

8. Legon W, Rowlands A, Opitz A, Sato TF, Tyler WJ: Pulsed ultrasound differentially stimulates somatosensory circuits in humans as indicated by EEG and FMRI. PLOS ONE 2012, 7:e51177.

9. Smits ES, Duraku LS, Niehof SP, Daanen HA, Hovius SE, Selles RW, Walbeehm ET: Cold-induced vasodilatation in cold-intolerant rats after nerve injury. J Plast Reconstr Aesthet Surg 2013, 66:1279-1286. 
10. Cutolo M, Sulli A, Pizzorni C, Smith V: Capillaroscopy as an outcome measure for clinical trials on the peripheral vasculopathy in SSc-is it useful? Int J Rheumatol 2010, 2010:784987.

11. Jan YK, Shen S, Foreman RD, Ennis WJ: Skin blood flow response to locally applied mechanical and thermal stresses in the diabetic foot. Microvasc Res 2013, 89:40-46.

12. Lin C-Y, Lin M-H, Cheung W-M, Lin T-N, Chen J-H, Chang C: In vivo cerebromicrovasculatural visualization using 3D [Delta]R2-based microscopy of magnetic resonance angiography (3D[Delta]R2-mMRA). Neuroimage 2009, 45:824-831.

13. Hillman EMC: Optical brain imaging in vivo: techniques and applications from animal to man. J Biomed Opt 2007, 12:051402-051428.

14. Tsytsarev V, Bernardelli C, Maslov Kl: Living brain optical imaging: technology, methods and applications. J Neurosci Neuroeng 2012, 1:180-192.

15. Malonek D, Grinvald A: Interactions between electrical activity and cortical microcirculation revealed by imaging spectroscopy: implications for functional brain mapping. Science 1996, 272:551-554.

16. Prahl S: Optical Spectra. 2007. Available: http://omlc.ogi.edu/.

17. Wang LV, Wu H-i: Biomedical Optics: Principles and Imaging. Wiley; 2007. ISBN 978-0-471-74304-0.

18. Liao LD, Tsytsarev V, Delgado-Martinez I, Li ML, Erzurumlu R, Vipin A, Orellana J, Lin YR, Lai HY, Chen YY, Thakor NV: Neurovascular coupling: in vivo optical techniques for functional brain imaging. Biomed Eng Online 2013, 12:38.

19. Miao P, Li N, Thakor NV, Tong S: Random process estimator for laser speckle imaging of cerebral blood flow. Opt Express 2010, 18:218-236.

20. Li N, Jia X, Murari K, Parlapalli R, Rege A, Thakor NV: High spatiotemporal resolution imaging of the neurovascular response to electrical stimulation of rat peripheral trigeminal nerve as revealed by in vivo temporal laser speckle contrast. J Neurosci Methods 2009, 176:230-236.

21. Peng M, Rege A, Nan L, Thakor NV, Shanbao T: High resolution cerebral blood flow imaging by registered laser speckle contrast analysis. IEEE Trans Biomed Eng 2010, 57:1152-1157.

22. Culver JP, Siegel AM, Franceschini MA, Mandeville JB, Boas DA: Evidence that cerebral blood volume can provide brain activation maps with better spatial resolution than deoxygenated hemoglobin. Neuroimage 2005, 27:947-959.

23. Liao L-D, Li M-L, Lai H-Y, Shih Y-YI, Lo Y-C, Tsang S, Chao PC-P, Lin C-T, Jaw F-S, Chen Y-Y: Imaging brain hemodynamic changes during rat forepaw electrical stimulation using functional photoacoustic microscopy. Neuroimage 2010, 52:562-570.

24. Zhang HF, Maslov K, Wang LV: In vivo imaging of subcutaneous structures using functional photoacoustic microscopy. Nat Protoc 2007, 2:797-804.

25. Ermilov SA, Khamapirad T, Conjusteau A, Leonard MH, Lacewell R, Mehta K, Miller T, Oraevsky AA: Laser optoacoustic imaging system for detection of breast cancer. J Biomed Opt 2009, 14:024007.

26. Kircher MF, de la Zerda A, Jokerst JV, Zavaleta CL, Kempen PJ, Mittra E, Pitter K, Huang R, Campos C, Habte F, Sinclair R, Brennan CW, Mellinghoff IK, Holland EC, Gambhir SS: A brain tumor molecular imaging strategy using a new triple-modality MRI-photoacoustic-Raman nanoparticle. Nat Med 2012, 18:829-834.

27. Liao L-D, Lin C-T, Shih Y-YI, Lai H-Y, Zhao W-T, Duong TQ, Chang J-Y, Chen Y-Y, Li M-L: Investigation of the cerebral hemodynamic response function in single blood vessels by functional photoacoustic microscopy. J Biomed Opt 2012, 17:061210-10.

28. Hu S, Wang LV: Neurovascular photoacoustic tomography. Front Neuroenerg 2010, 2:12.

29. Konstas AA, Choi JH, Pile-Spellman J: Neuroprotection for ischemic stroke using hypothermia. Neurocrit Care 2006, 4:168-178.

30. Rupp A, Dornseifer U, Fischer A, Schmahl W, Rodenacker K, Jutting U, Gais P, Biemer E, Papadopulos N, Matiasek K: Electrophysiologic assessment of sciatic nerve regeneration in the rat: surrounding limb muscles feature strongly in recordings from the gastrocnemius muscle. J Neurosci Methods 2007, 166:266-277.

31. Stein EW, Maslov K, Wang LV: Noninvasive, in vivo imaging of blood-oxygenation dynamics within the mouse brain using photoacoustic microscopy. J Biomed Opt 2009, 14:020502

32. Li L, Zemp RJ, Lungu G, Stoica G, Wang LV: Photoacoustic imaging of lacZ gene expression in vivo. J Biomed Opt 2007, 12:020504

33. Liao LD, Lin CT, Shih YYI, Duong TQ, Lai HY, Wang PH, Wu R, Tsang S, Chang JY, Li ML: Transcranial imaging of functional cerebral hemodynamic changes in single blood vessels using in vivo photoacoustic microscopy. J Cereb Blood Flow Metab 2012, 32:938-951.

34. Liao L-D, Lin C-T: Novel trends in biosensors used for electroencephalography measurements in neurocognitive engineering applications. J Neurosci Neuroeng 2012, 1:1006.

35. Duraku LS, Smits ES, Niehof SP, Hovius SE, Walbeehm ET, Selles RW: Thermoregulation in peripheral nerve injury-induced cold-intolerant rats. J Plast Reconstr Aesthet Surg 2012, 65:771-779.

36. Kusters FJ, Walbeehm ET, Niehof SP: Neural influence on cold induced vasodilatation using a new set-up for bilateral measurement in the rat hind limb. J Neurosci Methods 2010, 193:100-105.

37. Suichies HE, Aarnoudse JG, Wouda AA, Jentink HW, de Mul FF, Greve J: Digital blood flow in cooled and contralateral finger in patients with Raynaud's phenomenon. Comparative measurements between photoelectrical plethysmography and laser Doppler flowmetry. Angiology 1992, 43:134-141.

38. Willie CK, Ainslie PN: Cool head, hot brain: cerebral blood flow distribution during exercise. J Physio/ 2011, 589:2657-2658.

39. Seiyama A, Seki J, Tanabe H, Ooi Y, Satomura Y, Fujisaki H, Yanagida T: Regulation of oxygen transport during brain activation: stimulus-induced hemodynamic responses in human and animal cortices. Dynamic Medicine 2003, 2:6.

40. Hirano Y, Stefanovic B, Silva AC: Spatiotemporal evolution of the functional magnetic resonance imaging response to ultrashort stimuli. J Neurosci 2011, 31:1440-1447.

41. Nemoto M, Sheth S, Guiou M, Pouratian N, Chen JWY, Toga AW: Functional signal- and paradigm-dependent linear relationships between synaptic activity and hemodynamic responses in rat somatosensory cortex. J Neurosci 2004, 24:3850-3861. 
42. Ogawa S, Lee TM, Kay AR, Tank DW: Brain magnetic resonance imaging with contrast dependent on blood oxygenation. Proc Natl Acad Sci 1990, 87:9869-9872.

43. Schridde U, Khubchandani M, Motelow JE, Sanganahalli B, Hyder F: Negative BOLD with large increases in neuronal activity. Cereb Cortex 2008, 18:1814-1827.

44. Georg Royl MF, Christoph L, Dirk M, Nikolas O, Jens S, Matthias K-B, Ulrich D, Ute L: Hypothermia effects on neurovascular coupling and cerebral metabolic rate of oxygen. Neuroimage 2008, 40:1523-1532.

doi:10.1186/1475-925X-12-120

Cite this article as: Liao et al: Imaging of temperature dependent hemodynamics in the rat sciatic nerve by functional photoacoustic microscopy. BioMedical Engineering OnLine 2013 12:120.

\section{Submit your next manuscript to BioMed Central} and take full advantage of:

- Convenient online submission

- Thorough peer review

- No space constraints or color figure charges

- Immediate publication on acceptance

- Inclusion in PubMed, CAS, Scopus and Google Scholar

- Research which is freely available for redistribution

Submit your manuscript at www.biomedcentral.com/submit

() Biomed Central 